\title{
Encefalopatía inducida por 5-fluorouracilo Un mismo agente, dos entidades diferentes
}

\section{Encephalopathy induced by 5-fluorouracil}

\author{
The same agent, two different entities
}

\author{
Juan Carlos Velásquez, Diego Mauricio González, \\ Paola Andrea Pinilla, César Andrés Rodríguez, Ricardo Elías Brugés \\ - Bogotá, D.C. (Colombia)
}

\section{Resumen}

La neurotoxicidad de los medicamentos antineoplásicos es un fenómeno de relativa frecuencia, que ocurre principalmente sobre el sistema nervioso periférico y más comúnmente con fármacos citotóxicos como platinos, taxanos y derivados de la vinca. La toxicidad sobre el sistema nervioso central asociado al uso del 5-fluorouracilo es por el contrario un evento poco común, pero no por ello menos importante. El clínico debe aprender a reconocer y tratar las dos variedades de esta condición médica: la encefalopatía aguda y la leucoencefalopatía subaguda; cada una con fisiopatología, manifestaciones clínicas y tratamiento diferentes. La siguiente presentación de casos ejemplifica estas dos variedades y brinda elementos diagnósticos y terapéuticos para cada una de ellas. (Acta Med Colomb 2015; 40: 338-344).

Palabras clave: fluorouracilo, leucoencefalopatías, toxicidad, hiperamonemia, deficiencia de tiamina.

\begin{abstract}
The neurotoxicity of anticancer drugs is a relatively common phenomenon, occurring mainly on the peripheral nervous system, most commonly with cytotoxic drugs such as platinum, taxanes and vinca derivatives. Toxicity on the central nervous system associated with the use of 5-fluorouracil is on the contrary a rare event, which does not make it less important. The clinician must learn to recognize and treat the two varieties of this medical condition: acute encephalopathy and sub-acute leukoencephalopathy, each with different pathophysiology, clinical manifestations and treatment. The following case presentation illustrates these two varieties and provides diagnostic and therapeutic elements for each one of them. (Acta Med Colomb 2015; 40: 338-344).
\end{abstract}

Keywords: fluorouracil, leukoencephalopathies, toxicity, hyperammonemia, thiamine deficiency.

Dres. Juan Carlos Velásquez, Diego Mauricio González, Paola Andrea Pinilla: Especialistas en entrenamiento en Oncología Clínica; Dr. César Andrés Rodríguez: Especialista en Neuroradiología; Dr. Ricardo Elías Brugés: Oncólogo clínico, Coordinador Grupo de Oncología Clínica. Instituto Nacional de Cancerología, Bogotá, D. C. (Colombia).

Correspondencia. Dr. Juan Carlos Velásquez E-mail: jcvelasquez.2000@gmail.com Recibido: 19/IV/2015 Aceptado: 21/X/2015

\section{Introducción}

La toxicidad de los antineoplásicos sobre el sistema nervioso es un evento adverso relativamente frecuente que afecta la calidad de vida de los pacientes con cáncer y aún en ciertas ocasiones la continuidad de su tratamiento. Si bien la mayoría de casos se concentra en el sistema nervioso periférico, donde puede afectar hasta $38 \%$ de los pacientes con terapias citotóxicas múltiples, el compromiso central puede ser más importante, ocasionando convulsiones, alteraciones cognitivas, deterioro de la conciencia y encefalopatía entre otros (1-3). El 5-fluorouracilo (5-FU), derivado pirimidínico fluorinado ampliamente usado en el tratamiento de tumores gastrointestinales y de cabeza y cuello, tiene un perfil de toxicidad principalmente dirigido al aparato digestivo (estomatitis, náuseas, vómito, diarrea, etc.), a la médula ósea (leucopenia y anemia) y a la piel (síndrome mano-pie), siendo mucho menos frecuentes la toxicidad cardiovascular y la neurológica (4). A continuación describimos dos casos de encefalopatía asociada al uso de 5-FU, que ilustran las dos variedades principales de la neurotoxicidad por este medicamento.

\section{Caso 1}

Paciente de 57 años de edad de sexo femenino, con diagnóstico de adenocarcinoma de recto inferior estado IIIC T3N2bM0 moderadamente diferenciado, desde mayo de 2010. En octubre de 2012 presentó una recaída ganglionar retroperitoneal y parenquimatosa pulmonar y se inicia pri- 
mera línea de tratamiento para enfermedad metastásica con esquema CapeOX (capecitabina y oxaliplatino) recibiendo nueve ciclos, tras lo cual presenta progresión retroperitoneal. Se prescribe una segunda línea de quimioterapia con FOLFIRI (5-FU más irinotecan) y bevacizumab. En el segundo día del primer ciclo de quimioterapia, presentó confusión y somnolencia, y unas horas más tarde una convulsión tónico clónica generalizada, disartria, estupor, hipotonía de las cuatro extremidades, y nistagmus horizontal, en ausencia de signos meníngeos, hipertensión, fiebre o focalización motora. Recibió fenitoína y oxígeno, se suspendió la quimioterapia y se tomaron paraclínicos. La química sanguínea (glucemia, electrolitos y las pruebas de función hepática), así como el hemograma fueron normales, salvo leve elevación de la creatinina ya conocida de antemano. La resonancia cerebral (RNM) contrastada fue normal (Figura 1). El líquido cefalorraquídeo no mostró anormalidades. Se descartó proceso infeccioso. Con sospecha de encefalopatía aguda por 5-FU se solicitaron niveles de amonio que resultaron elevados $(52 \mu \mathrm{mol} / \mathrm{L}$; valores normales entre $9 \mathrm{y}$ $30 \mu \mathrm{mol} / \mathrm{L}$ ) y se inició tratamiento con lactulosa por sonda nasogástrica. Se administró tiamina (500 mg IV al día por cinco días). Al quinto día empezó a notarse mejoría del estado de conciencia, pasando por leve agitación, delirio, incoordinación motora y ataxia temporales con posterior recuperación de la disartria, el nistagmus y el curso y contenido del pensamiento. Una semana después del inicio del cuadro, sus síntomas habían desaparecido. La paciente no quiso continuar ningún tipo de quimioterapia y fue enviada a casa para mejor cuidado de soporte.

\section{Caso 2}

Paciente femenina de 49 años con adenocarcinoma gástrico de tipo difuso (células en anillo de sello) ulcerado, con componente mucoproductor y carcinomatosis peritoneal (estadio IV T4aNxM0) diagnosticado en junio de 2013, quien inició tratamiento con 5-FU y cisplatino; luego del segundo ciclo, sin embargo, se suspendió por mucositis severa. Retomó sus controles tres meses después (febrero
2014) documentándose progresión ovárica, hepática y ascitis. Recibió oxaliplatino y capecitabine, sin embargo en el segundo ciclo presentó un primer episodio de disartria, dismetría y ataxia por lo que acudió a urgencias donde se obtuvo una química sanguínea sin alteraciones descartando causas vasculares, metabólicas o infecciosas. La RNM cerebral mostró leucoencefalopatía simétrica bilateral, comprometiendo la sustancia blanca profunda y el cuerpo calloso, con mayor alteración del esplenio, asociada a restricción de movimiento del agua en las secuencias de difusión y en el mapa ADC (apparent diffusion coefficient) atribuibles a vacuolización de la mielina (Figuras 2a y 2b). Ante el hallazgo imagenológico sugestivo de toxicidad por quimioterapia y dado que la paciente mostró recuperación de manera espontánea durante la observación, se decidió suspender este esquema terapéutico. Se prescribió docetaxel, sin embargo presentó progresión con ascitis, por lo cual se cambió nuevamente su terapia a carboplatino + 5-FU. Dos semanas después de la primera dosis, la paciente ingresó a urgencias presentando esta ocasión un mayor compromiso del estado de conciencia con somnolencia, disartria y disminución simétrica de la fuerza en las cuatro extremidades, de predominio distal. Al igual que en su hospitalización de febrero 2014 se descartaron trastornos hidroelectrolíticos asociados, infección o trastorno metabólico. Se realizó una RNM que mostró una extensa leucoencefalopatía simétrica bilateral, comprometiendo la sustancia blanca profunda y el cuerpo calloso con mayor afectación del esplenio, lo que se asocia a restricción al movimiento del agua en las secuencias de difusión y en el mapa ADC atribuibles a vacuolización de la mielina (Figuras $2 \mathrm{c}$ y $2 \mathrm{~d}$ ). A diferencia del primer episodio, los cambios en el estado de conciencia no se modificaron espontáneamente. Ante la sospecha de toxicidad por fluorinados se midieron los niveles de amonio y resultaron normales. Se suspendieron los antineoplásicos, se administró tiamina y otras medidas de soporte, pero no hubo cambios su condición clínica. Tres semanas después es dada de alta sin recuperación de su condición motora (Barthel de 5 puntos y ECOG 4).
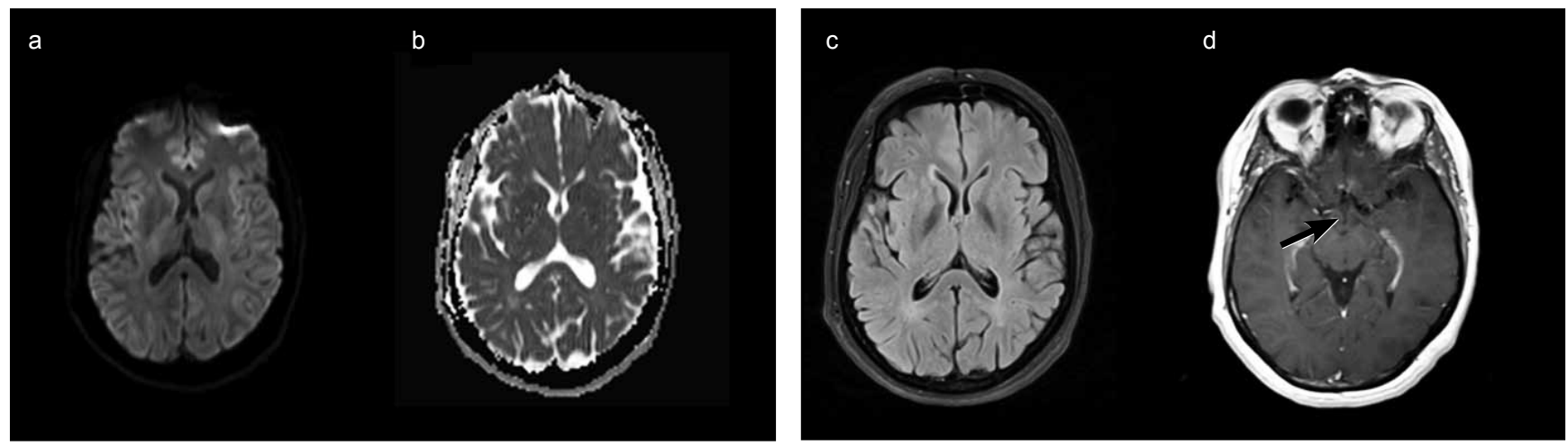

Figura 1. Resonancia nuclear magnética cerebral de la paciente del caso $N^{o} 1$. Paciente de 57 años de edad adenocarcinoma de recto metastásico en manejo quimioterapéutico con $5-$ fluorouracilo con confusión, somnolencia, y convulsión tónico clónica generalizada. (a) Secuencia de difusión (DWI) y (b) Mapa de coeficiente de difusión aparente (ADC) que no demuestran áreas de restricción al movimiento del agua. (c) Imagen potenciada en T2 FLAIR que muestran adecuada configuración de la sustancia blanca profunda y de los núcleos grises de la base. (d) Secuencia axial potenciada en T1 con gadolinio que evidencia los cuerpos mamilares de configuración normal (flecha negra). 
a

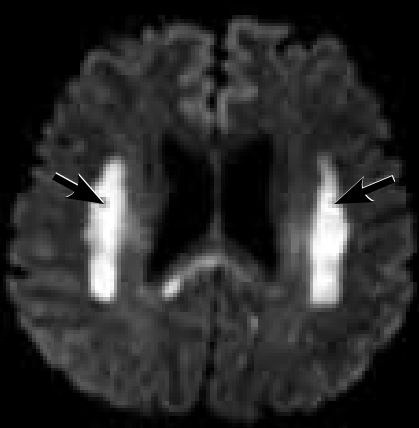

b

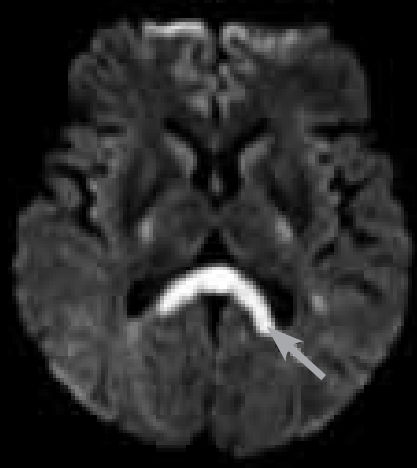

c

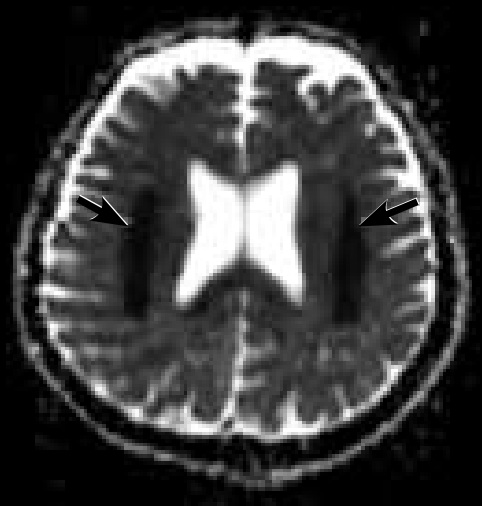

d

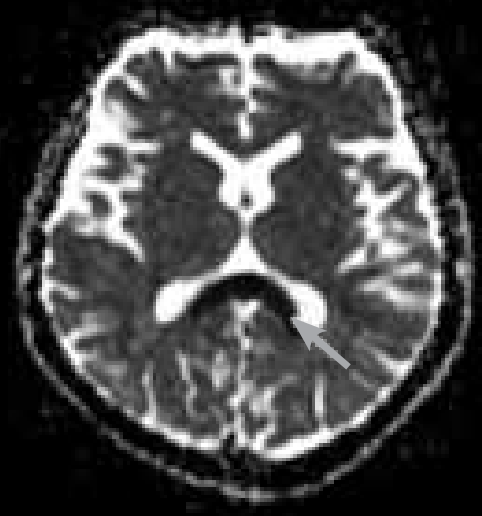

e

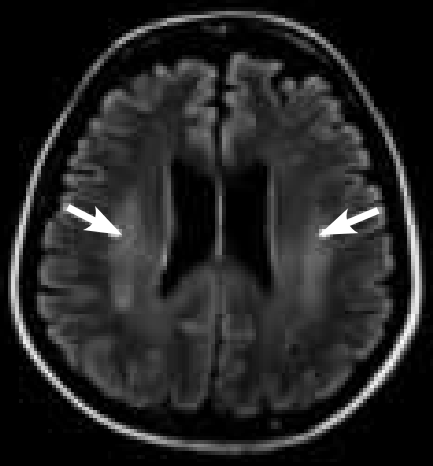

f



Figura 2. Cambios imagenológicos en la RNM en paciente con leucoencefalopatía aguda. Paciente de 49 años de edad con adenocarcinoma gástrico metastásico quien recibe manejo quimioterapéutico con 5-Fluorouracilo. Presenta cuadro de somnolencia, disartria y disminución simétrica de la fuerza en las 4 extremidades. (a, b) Secuencias de difusión (DWI) y (c, d) Mapa de coeficiente de difusión aparente ( $A D C$ ) que muestran restricción al movimiento del agua en la sustancia blanca profunda de ambos centros semiovales (flechas negras) y en el esplenio del cuerpo calloso (flechas grises). (e,f) Imágenes potenciadas en T2 FLAIR que muestran aumento de su intensidad en las mismas regiones por leucoencefalopatía (flechas blancas). 


\section{Discusión}

La neurotoxicidad por 5-FU es un evento poco frecuente en la práctica clínica. El primer caso fue informado en 1964 por Riehl y Brown, quienes describieron un paciente con síndrome cerebeloso agudo asociado al uso de este medicamento (5). Desde entonces periódicamente han aparecido reportes de casos en la literatura, bien ocurridos durante su administración o bien días o semanas después (6-8).

Clásicamente, se han descrito dos formas de toxicidad: una aguda y una tardía (6); la primera consiste en un cuadro de encefalopatía (confusión, letargia, somnolencia, estupor y convulsiones) así como de manifestaciones cerebelosas (ataxia, incoordinación, alteraciones de la mirada conjugada, nistagmus, etc.) que corresponden a lo ilustrado por nuestro primer caso $(9,10)$; su frecuencia es de $2-4 \%(5)$. La segunda es también poco frecuente $(1.3-5 \%)(11,12)$, se caracteriza por leucoencefalopatía subaguda multifocal que puede desarrollarse semanas o meses y tiene como signos el inicio subagudo de anormalidades cognoscitivas y de conciencia, disartria, disfagia, afasia, debilidad focal y ataxia de las extremidades y de la marcha, e inclusive tetraplejía, como en nuestro segundo caso $(11,13)$.

La neurotoxicidad por 5-FU puede producirse tanto con infusiones endovenosas como con los bolos, con dosis altas o con intermedias. Usualmente no está asociada a compromiso hematológico o gastrointestinal (salvo en los casos de deficiencia de dihidropirimidina deshidrogenasa -DPD-) y puede ser o no reversible tras la descontinuación del medicamento $(6,14)$.

El mecanismo de la toxicidad aguda del 5-FU no ha sido aclarado por completo. Es posible que se deba a la acción del fluoroacetato, un metabolito del 5-FU que inhibe el ciclo de Krebs (15). Como consecuencia de este fenómeno se produce acidosis láctica y un bloqueo secundario del ciclo de la urea, que induce acumulación de amonio y por ello la neurotoxicidad (Figura 3) (16). Otra teoría sostiene que el 5-FU incrementa el metabolismo celular de tiamina, precipitando una deficiencia aguda de esta vitamina (17) o exacerbando una carencia previa (18). La tiamina pirofosfato es la forma activa y sirve de cofactor en diferentes procesos metabólicos como la conversión de piruvato a acetil coenzima A y la de alfa- cetoglutarato a succinil coenzima A en el ciclo del ácido cítrico, así como varias reacciones en el ciclo de las pentosas (18). La formación de tiamina pirofosfato a partir de tiamina, es bloqueada por el 5-FU. La ataxia, el nistagmus, la confusión mental y los cambios cognitivos en estos pacientes, similares a los de la encefalopatía de Wernicke $(7,8)$ y su resolución tras el uso de tiamina $(8$, $14,18)$ refuerzan esta teoría.

El mecanismo de neurotoxicidad tardía tampoco se conoce con precisión; no se sabe si comparte los mismos procesos de la forma aguda, pero es probable que sean diferentes, más de tipo inmune e inflamatorio (13). Las imágenes de RNM sugieren desmielinización y vacuolización de la mielina que en los estudios histopatológicos corresponden a fragmentación de fibras axonales, alteración mielínica y macrófagos dispersos (19). También se han descrito depósitos de interleuquina 1-alfa, interleuquina 6 y factor de necrosis tumoral (20). Otros estudios, por el contrario, sugieren un daño citotóxico directo inducido por el 5-FU en la neurona, provocando un aumento de la apoptosis (21).

Nuestro primer caso cumplió con los criterios diagnósticos para encefalopatía aguda relacionada con 5-FU (6): a) desarrollo de la encefalopatía durante de la administración

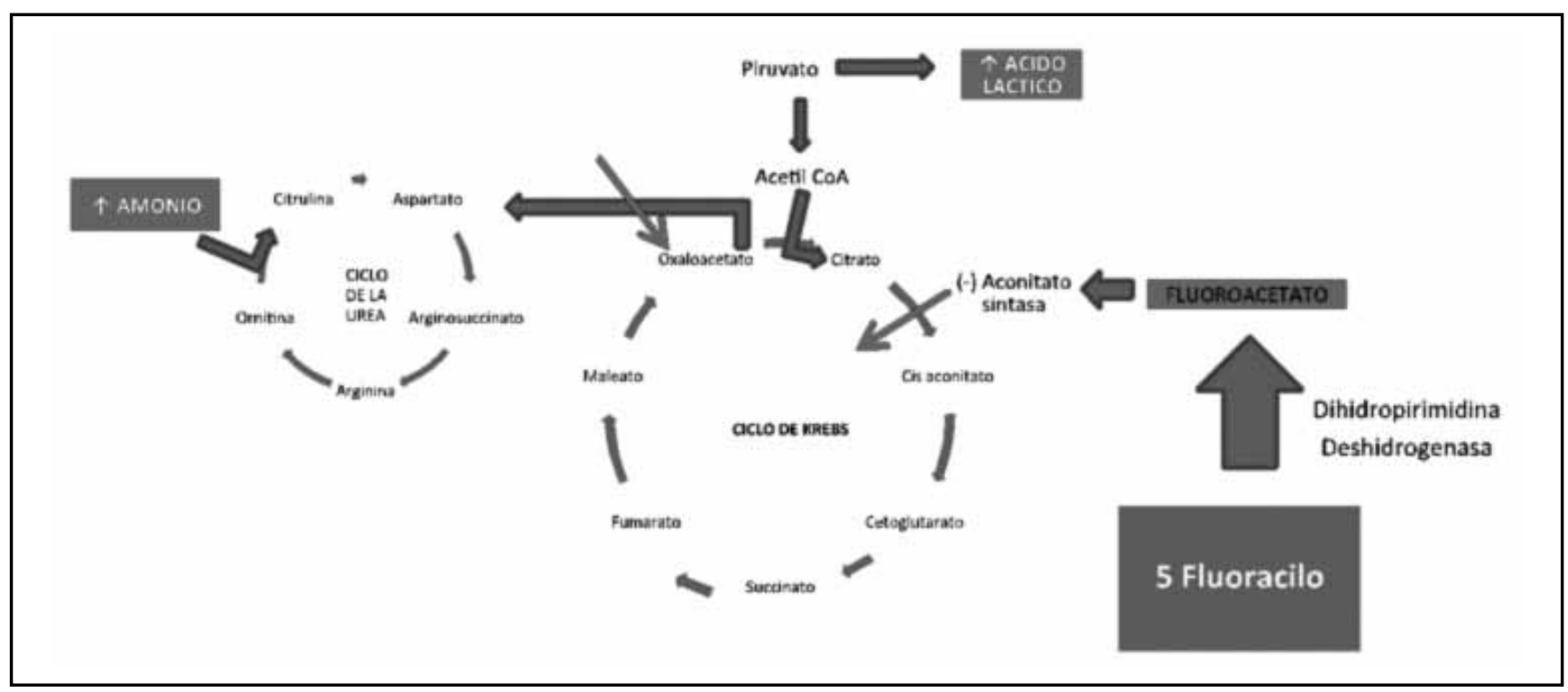

Figura 3. Efectos del 5-FU en el ciclo de Krebs y en el ciclo de la urea. El 5 FU se convierte en fluoroacetato por acción de la DPD. El fluoroacetato inhibe la aconitatosintasa lo cual disminuye la producción de cis-aconitato y por último de oxaloacetato. La falta de oxaloacetato genera un bloqueo del ciclo de la urea con la consecuente acumulación de amonio. El bloqueo del ciclo de Krebs produce a su vez una acumulación de piruvato y acidosis láctica. 
del 5-FU poco después; b) exclusión de otros factores metabólicos que puedan afectar la conciencia y el funcionamiento mental, como hipoglucemia, falla orgánica, alteración de los electrolitos, sepsis y compromiso del sistema nervioso central por cáncer; y c) exclusión de un efecto adverso por medicamentos concomitantes. Los exámenes paraclínicos revelaron la hiperamonemia característica en estos pacientes (22-24); algunos pueden tener acidosis láctica (16) y grados variables de neutropenia o trombocitopenia según haya o no toxicidad hematológica concomitante. El líquido cefalorraquídeo suele ser normal y en la forma aguda las neuroimágenes, característicamente no revelan cambios, salvo en algunos casos compatibles con encefalopatía de Wernicke donde la RNM muestra aumento de la intensidad de señal en las secuencias potenciadas en T2 (T2 y FLAIR o fluid attenuated inversión recovery) en la sustancia gris periacueductal del mesencéfalo (8), en la región medial de los tálamos, el hipotálamo y la corteza del cerebelo así como realce posterior a la administración endovenosa de gadolinio en los cuerpos mamilares (25). La sensibilidad y especificidad de la RNM están alrededor de 53 y $93 \%$ respectivamente (8).

En la neurotoxicidad tardía (leucoencefalopatía) lo típico es encontrar algunas hipodensidades en la sustancia blanca hemisférica en la tomografía e hiperintensidad periventricular en la RNM debido a desmielinización multifocal $(11,26)$ como se pudo apreciar en nuestro segundo caso. Las imágenes de difusión, suelen mostrar restricción en la sustancia blanca profunda en forma bilateral, así como en el esplenio del cuerpo calloso (12); junto a hiperintensidad en las secuencias potenciadas en T2 FLAIR (27). Estos hallazgos se deben a la separación segmentaria de las lamelas de mielina por disociación de las líneas intraperiódicas, con vacuolización y edema, sin cambios axonales o isquemia; la compartimentalización de las moléculas de agua dentro de las vacuolas resulta en la restricción de la difusión.

En cuanto a la evolución del cuadro, la duración de la encefalopatía varía de días a semanas, generalmente con resolución completa $(24,28)$, pero se han descrito alteraciones más prolongadas o aun secuelas permanentes, así como casos fatales. La leucoencefalopatía desmielinizante por su parte, suele tener una evolución más lenta, muchas veces un comportamiento crónico y aún irreversible (11), si bien algunos pacientes logran la resolución clínica a los pocos días (6). En los pacientes con deficiencia de DPD suele haber gran toxicidad hematológica y gastrointestinal, lo cual no se apreció en ninguno de nuestros casos y por ello no se consideró probable. La Tabla 1 muestra una comparación de los hallazgos más frecuentes en las dos formas de neurotoxicidad por 5-FU.

En la evaluación de nuestros pacientes consideramos algunos diagnósticos diferenciales. Las manifestaciones exhibidas en uno y otro no correspondían a lo observado en el síndrome de leucoencefalopatía posterior reversible (PRES, por sus siglas en inglés). Esta entidad, descrita inicialmente por Hinchey y colaboradores en 1996 (29), se caracteriza por una alteración de la esfera mental, desórdenes de la visión, cefalea, convulsiones y por un típico edema

Tabla 1. Elementos para el diagnóstico de encefalopatía por 5-FU. Principales características de los dos tipos de neurotoxicidad sobre el sistema nervioso central producida por 5 - fluorouracilo.

\begin{tabular}{|c|c|c|}
\hline Características & Encefalopatía aguda & Leucoencefalopatía subaguda \\
\hline Principales signos y síntomas & $\begin{array}{l}\text { - Confusión, letargia, } \\
\text { - Somnolencia, estupor, coma } \\
\text { - Convulsiones } \\
\text { - Ataxia } \\
\text { - Dismetría } \\
\text { - Alteraciones de la mirada conjugada } \\
\text { - Incoordinación motora } \\
\text { - Nistagmus }\end{array}$ & 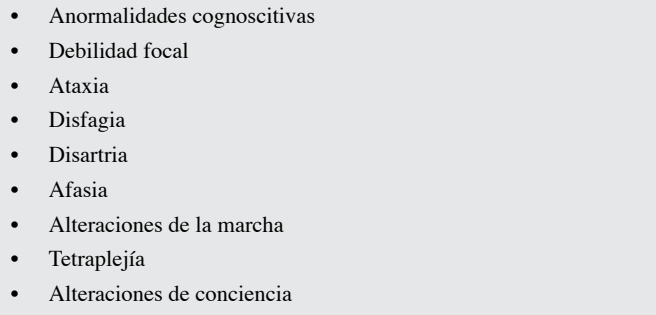 \\
\hline Criterios para el diagnóstico & \multicolumn{2}{|c|}{$\begin{array}{l}\text { - Desarrollo de la encefalopatía durante la administración del 5-FU o poco tiempo después } \\
\text { - Exclusión de otros factores metabólicos que puedan explicar los síntomas } \\
\text { - Exclusión de un efecto adverso por otros medicamentos concomitantes }\end{array}$} \\
\hline Exámenes paraclínicos & $\begin{array}{l}\text { - Descartar alteraciones metabólicas (hipoglucemia, uremia, } \\
\text { desórdenes hidroelectrolíticos, encefalopatía hepática) } \\
\text { - Hiperamonemia } \\
\text { - Acidosis láctica } \\
\text { - Puede haber o no toxicidad hematológica (¿deficiencia de DPD?) }\end{array}$ & $\begin{array}{l}\text { - Descartar alteraciones metabólicas (hipoglucemia, uremia, desórdenes } \\
\text { hidroelectrolíticos, encefalopatía hepática) } \\
\text { - Puede haber o no toxicidad hematológica } \\
\text { (¿deficiencia de DPD?) }\end{array}$ \\
\hline $\begin{array}{l}\text { Hallazgos en neuroimágenes } \\
\text { (RNM) }\end{array}$ & $\begin{array}{l}\text { - Normal } \\
\text { Puede haber hiperintensidad en T2 en regiones periventriculares } \\
\text { del tálamo, hipotálamo, cuerpos mamilares, piso del IV } \\
\text { ventrículo y porción medial del cerebelo }\end{array}$ & $\begin{array}{l}\text { - Hiperintensidad periventricular (leucoencefalopatía). } \\
\text { - En la difusión, restricción (hiperintensidad) a nivel frontal y parietal } \\
\text { en la sustancia blanca bilateral) } \\
\text { - En el FLAIR: hiperintensidad en las mismas zonas }\end{array}$ \\
\hline
\end{tabular}


subcortical cerebral de predominio posterior, el cual puede comprometer también el cerebelo. Este síndrome, puede producirse por múltiples causas, incluyendo el uso de agentes de quimioterapia, y se han descrito casos relacionados con gemcitabina (30), cisplatino (31), oxaliplatino (32), bevacizumab (33), sunitinib (34), FOLFIRI/bevacizumab (35) y cisplatino/5-FU (36), entre otros. En nuestro conocimiento no se han informado episodios de PRES por 5-FUo irinotecan como agentes independientes, ni con FOLFIRI sin bevacizumab. El PRES implica siempre la presencia de edema vasogénico subcortical de predominio posterior, bilateral y simétrico que estuvo totalmente ausente en la RNM de nuestra primera paciente y en cuanto al segundo caso, nunca manifestó la cefalea global constante, intensa (37), ni las alteraciones visuales con hemianopsias, auras y/o alucinaciones $(29,38)$ que lo acompañan; más aún las alteraciones de la RNM no tenían predilección por las zonas posteriores como es frecuente en el PRES y sobre todo, su cuadro neurológico luego de tres semanas de evolución, no tuvo cambio alguno, lo cual no corresponde a la naturaleza generalmente benigna y transitoria de esta entidad.

Otro diagnóstico diferencial que debe tenerse presente por sus implicaciones terapéuticas es el de un evento isquémico del sistema nervioso central. En la literatura, se han descrito casos de pacientes con leucoencefalopatía asociada agentes de quimioterapia que se manifiestan de forma aguda, con signos focalización motora o sensitiva como hemiparesia, disfasia, hemihipoestesia, hemidisesesia, disfagia, dismetría y disfonía que semejan un evento isquémico (39). Estos casos son más frecuentes con metotrexate intratecal o intravenoso en dosis altas, pero también se han informado con 5-FU, pudiendo algunos de ellos cumplir incluso criterios de trombolisis, conduciendo a la administración errada de terapias de reperfusión (40). El diagnóstico se suele aclarar con la evolución clínica y radiológica, bien al progresar a un compromiso más difuso de ambos hemisferios, o bien al resolver sin secuelas, con una rápida normalización de las hipointensidades en las secuencias de coeficiente de difusión aparente (ADC por su nombre en inglés), que en isquemia por el contrario, cuando son marcadas, son sinónimo de mal pronóstico.

Por último, una posibilidad alterna a las anteriores en nuestra primera paciente, podría haber sido un efecto secundario al irinotecan, pero la neurotoxicidad de este agente es una condición muy rara y usualmente sus efectos adversos están más relacionados con neutropenia, diarrea, y síndrome colinérgico. Los casos de toxicidad en el sistema nervioso central por irinotecan suelen presentarse dentro de la primera hora de infusión del medicamento, su manifestación principal es la disartria y solo en algunos hay marcha atáxica y afasia motora, sin compromiso de conciencia (41).

En lo referente al tratamiento, nuestra primera paciente respondió a la administración de lactulosa como terapia contra la hiperamonemia $(22,28)$. Algunos autores han empleado neomicina, benzoato de sodio y fenilacetato de sodio, pero no se dispone de ensayos clínicos al respecto; los dos últimos convierten la glicina en hipurato y la glutamina en fenilacetato de glutamina respectivamente y tanto el hipurato como el fenilacetato de glutamina son formas de amonio diferentes a la úrea que pueden ser excretadas libremente por la orina. En casos refractarios al tratamiento médico, otro recurso que puede emplearse es la diálisis, bien sea como hemodiálisis intermitente o bien como hemofiltración arteriovenosa o venovenosa; ambos procedimientos producen una rápida disminución de los niveles de amonio y pueden ser utilizados hasta lograr valores más seguros, inferiores a 200 ìmol/L $(28,42)$. Para el tratamiento de la encefalopatía similar a Wernike, se recomienda la administración de tiamina, en dosis que pueden ir desde $50 \mathrm{mg}$ intramusculares (6), hasta $500 \mathrm{mg}$ intravenosos al día por cinco días (ésta última fue la dosis usada por nosotros en el tratamiento de nuestra primera paciente), seguidos de tiamina oral $60 \mathrm{mg}$ al día según la evolución (8). Finalmente, para los casos de leucoencefalopatía subaguda se han recomendado los corticoesteroides $(11,12,19)$.

\section{Conclusiones}

La neurotoxicidad por 5-FU es un evento adverso importante, con dos variedades principales de presentación: la encefalopatía aguda o la leucoencefalopatía subaguda. Los casos comentados, ilustran las dos formas de toxicidad y su diagnóstico requiere de un buen juicio clínico, el apoyo de algunos exámenes de laboratorio y una correcta interpretación de las imágenes a la luz del cortejo sintomático. La evolución de la enfermedad es variada y puede generar secuelas graves de carácter permanente. Es necesario avanzar en el entendimiento de la fisiopatología de estos fenómenos, en particular en el de la leucoencefalopatía subaguda, dado su carácter más permanente e incapacitante.

\section{Declaración de financiación y posibles conflictos de interés}

Los autores del presente artículo declaran que no tiene ningún conflicto de interés en relación con esta publicación. No hubo colaboración externa en la concepción diseño, redacción o presentación de este reporte. No hubo financiación de ningún tipo por fuentes externas a los autores.

\section{Referencias}

1. Jaggi AS, Singh N. Mechanisms in cancer-chemotherapeutic drugs-induced peripheral neuropathy. Toxicology. 2012; 291 (1-3): 1-9.

2. Carozzi VA, Canta A, Chiorazzi A, Cavaletti G. Chemotherapy-induced peripheral neuropathy: What do we know about mechanisms? Neurosci Lett. 2014.

3. Cavaletti G, Zanna C. Current status and future prospects for the treatment of chemotherapy-induced peripheral neurotoxicity. Eur J Cancer. 2002; 38 (14): 1832-7.

4. Alter P, Herzum M, Soufi M, Schaefer JR, Maisch B. Cardiotoxicity of 5-fluorouracil. Cardiovasc Hematol Agents Med Chem. 2006; 4 (1): 1-5.

5. Riehl JL, Brown WJ. Acute cerebellar syndrome secondary to 5-fluorouracil therapy. Neurology. 1964; 14: 961-7.

6. Pirzada NA, Ali, II, Dafer RM. Fluorouracil-induced neurotoxicity. Ann Pharmacother. 2000; 34 (1): 35-8

7. Kondo K, Fujiwara M, Murase M, Kodera Y, Akiyama S, Ito K, et al. Severe acute metabolic acidosis and Wernicke's encephalopathy following chemotherapy with 5-fluorouracil and cisplatin: case report and review of the literature. Jpn J Clin Oncol. 1996; 26 (4): 234-6. 
8. Cho IJ, Chang HJ, Lee KE, Won HS, Choi MY, Nam EM, et al. A case of Wernicke's encephalopathy following fluorouracil-based chemotherapy. J Korean Med Sci. 2009; 24 (4): 747-50.

9. Moertel CG, Reitemeier RJ, Bolton CF, Shorter RG. Cerebellar ataxia associated with fluorinated pyrimidine therapy. Cancer Chemother Rep. 1964; 41: 15-8.

10. Boileau G, Piro AJ, Lahiri SR, Hall TC. Cerebellar ataxia during 5-fluorouracil (NSC-19893) therapy. Cancer Chemother Rep. 1971; 55 (5): 595-8.

11. Luppi G, Zoboli A, Barbieri F, Crisi G, Piccinini L, Silingardi V. Multifocal leukoencephalopathy associated with 5-fluorouracil and levamisole adjuvant therapy for colon cancer. A report of two cases and review of the literature. The INTACC. Intergruppo Nazionale Terpia Adiuvante Colon Carcinoma. Ann Oncol . 1996; 7 (4): 412-5.

12. Paul BS, Singh G, Bansal R, Paul G. Diffusion weighted MR imaging of 5-fluorouracil and oxaliplatin-induced leukoencephalopathy. J Postgrad Med.2013; 59 (2): $135-7$.

13. Hook CC, Kimmel DW, Kvols LK, Scheithauer BW, Forsyth PA, Rubin $\mathbf{J}$, et al. Multifocal inflammatory leukoencephalopathy with 5-fluorouracil and levamisole. Ann Neurol. 1992; 31 (3): 262-7.

14. Kwon KA, Kwon HC, Kim MC, Kim SH, Oh SY, Lee S, et al. A case of 5-fluorouracil induced encephalopathy. Cancer Res Treat. 2010; 42 (2): 118-20.

15. Koenig H, Patel A. Biochemical basis for fluorouracil neurotoxicity. The role of Krebs cycle inhibition by fluoroacetate. Arch Neurol. 1970; 23 (2): 155-60.

16. Yeh KH, Cheng AL. High-dose 5-fluorouracil infusional therapy is associated with hyperammonaemia, lactic acidosis and encephalopathy. Br J Cancer. 1997; 75 (3): 464-5.

17. Aksoy M, Basu TK, Brient J, Dickerson JW. Thiamin status of patients treated with drug combinations containing 5-fluorouracil. Eur J Cancer. 1980; 16 (8): 1041-5.

18. Rosen A, van Kuilenburg A, Assmann B, Kuhlen M, Borkhardt A. Severe encephalopathy, lactic acidosis, vegetative instability and neuropathy with 5-Fluorouracil treatment-pyrimidine degradation defect or beriberi?. Case Rep Oncol. 2011; 4 (2): 371-6.

19. Choi SM, Lee SH, Yang YS, Kim BC, Kim MK, Cho KH. 5-fluorouracil-induced leukoencephalopathy in patients with breast cancer. J Korean Med Sci. 2001; 16 (3): 328-34.

20. Israel ZH, Lossos A, Barak V, Soffer D, Siegal T. Multifocal demyelinative leukoencephalopathy associated with 5-fluorouracil and levamisole. Acta Oncol. 2000; 39 (1): 117-20.

21. Han R, Yang YM, Dietrich J, Luebke A, Mayer-Proschel M, Noble M. Systemic 5 -fluorouracil treatment causes a syndrome of delayed myelin destruction in the central nervous system. J Biol. 2008; 7 (4): 12.

22. Kim YA, Chung HC, Choi HJ, Rha SY, Seong JS, Jeung HC. Intermediate dose 5-fluorouracil-induced encephalopathy. Jpn J Clin Oncol. 2006; 36 (1): 55-9.

23. Liaw CC, Liaw SJ, Wang CH, Chiu MC, Huang JS. Transient hyperammonemia related to chemotherapy with continuous infusion of high-dose 5-fluorouracil. Anticancer Drugs. 1993; 4 (3): 311-5.

24. Barrueco N, Such Diaz A, Saez de la Fuente J, Escobar I. [Hyperammonemic encephalopathy in a patient treated with 5-fluorouracil]. Farm Hosp. 2014; 38 (1): $77-8$.
25. Sechi G, Serra A. Wernicke's encephalopathy: new clinical settings and recent advances in diagnosis and management. Lancet Neurol. 2007; 6 (5): 442-55.

26. Murray CL, Ford WJ, Swenson KK, Heros D, Sperduto PW. Multifocal inflammatory leukoencephalopathy after fluorouracil and levamisole therapy for colon cancer. AJNR Am J Neuroradiol. 1997; 18 (8): 1591-2.

27. Bang M, Weon YC, Yoo HJ, Kwon JH. MR imaging in the early stage of 5-fluorouracil-induced leukoencephalopathy: DWI, MR perfusion, and MR spectroscopy. Clin Neurol Neurosurg. 2012; 114 (8): 1185-8.

28. Willson KJ, Nott LM, Broadbridge VT, Price T. Hepatic encephalopathy associated with cancer or anticancer therapy. Gastrointest Cancer Res. 2013; 6 (1): 11-6.

29. Hinchey J, Chaves C, Appignani B, Breen J, Pao L, Wang A, et al. A Reversible Posterior Leukoencephalopathy Syndrome. N Engl J Med. 1996; 334 (8): 494-500.

30. Rajasekhar A, George TJ, Jr. Gemcitabine-induced reversible posterior leukoencephalopathy syndrome: a case report and review of the literature. Oncologist. 2007; 12 (11): 1332-5.

31. Ito Y,Arahata Y, Goto Y, Hirayama M, Nagamutsu M, Yasuda T, et al. Cisplatin neurotoxicity presenting as reversible posterior leukoencephalopathy syndrome. AJNR Am J Neuroradiol. 1998; 19 (3): 415-7.

32. Pinedo DM, Shah-Khan F, Shah PC. Reversible Posterior Leukoencephalopathy Syndrome Associated With Oxaliplatin. J Clin Oncol. 2007; 25 (33): 5320-1.

33. Glusker P, Recht L, Lane B. Reversible posterior leukoencephalopathy syndrome and bevacizumab. N Engl J Med. 2006; 354 (9): 980-2; discussion -2.

34. Martín G, Bellido L, Cruz JJ. Reversible Posterior Leukoencephalopathy Syndrome Induced by Sunitinib. J Clin Oncol. 2007; 25 (23): 3559.

35. Allen JA, Adlakha A, Bergethon PR. Reversible posterior leukoencephalopathy syndrome after bevacizumab/FOLFIRI regimen for metastatic colon cancer. Arch Neurol. 2006; 63 (10): 1475-8.

36. Paul F, Aktas O, Dieste FJ, Kreitsch P, Vogel HP, Zipp F. [Relapsing reversible posterior leukoencephalopathy after chemotherapy with cisplatin and 5-fluorouracil]. Nervenarzt. 2006; 77 (6): 706-10.

37. Stott VL, Hurrell MA, Anderson TJ. Reversible posterior leukoencephalopathy syndrome: a misnomer reviewed. Intern Med J. 2005; 35 (2): 83-90.

38. Lysandropoulos AP, Rossetti AO. Postictal cortical visual impairment: a symptom of posterior reversible encephalopathy. Epilepsy Behav. 2010; 17 (2): 276-7.

39. Baehring JM, Fulbright RK. Delayed leukoencephalopathy with stroke-like presentation in chemotherapy recipients. J Neurol Neurosurg Psychiatry. 2008; 79 (5): 535-9.

40. Kinno R, Kii Y, Uchiyama M, Owan Y, Yamazaki T, Fukui T. 5-fluorouracilinduced leukoencephalopathy with acute stroke-like presentation fulfilling criteria for recombinant tissue plasminogen activator therapy. J Stroke Cerebrovasc Dis. 2014; 23 (2): 387-9.

41. Hamberg P, De Jong FA, Brandsma D, Verweij J, Sleijfer S. Irinotecan-induced central nervous system toxicity. Report on two cases and review of the literature. Acta Oncol. 2008; 47 (5): 974-8.

42. Nott L, Price TJ, Pittman K, Patterson K, Fletcher J. Hyperammonemia encephalopathy: an important cause of neurological deterioration following chemotherapy. Leuk Lymphoma. 2007; 48 (9): 1702-11. 